\title{
An observational cohort study investigating risk of malnutrition using the Malnutrition Universal Screening Tool in patients with stroke.
}

DOI:

10.1016/j.jstrokecerebrovasdis.2019.104405

\section{Document Version \\ Accepted author manuscript}

Link to publication record in Manchester Research Explorer

\section{Citation for published version (APA):}

Sremanakova, J., Burden, S., Kama, Y., Gittins, M., Lal, S., Smith, C., \& Hamdy, S. (2019). An observational cohort study investigating risk of malnutrition using the Malnutrition Universal Screening Tool in patients with stroke. Journal of Stroke and Cerebrovascular Diseases.

https://doi.org/10.1016/j.jstrokecerebrovasdis.2019.104405

\section{Published in:}

Journal of Stroke and Cerebrovascular Diseases

\section{Citing this paper}

Please note that where the full-text provided on Manchester Research Explorer is the Author Accepted Manuscript or Proof version this may differ from the final Published version. If citing, it is advised that you check and use the publisher's definitive version.

\section{General rights}

Copyright and moral rights for the publications made accessible in the Research Explorer are retained by the authors and/or other copyright owners and it is a condition of accessing publications that users recognise and abide by the legal requirements associated with these rights.

\section{Takedown policy}

If you believe that this document breaches copyright please refer to the University of Manchester's Takedown Procedures [http://man.ac.uk/04Y6Bo] or contact uml.scholarlycommunications@manchester.ac.uk providing relevant details, so we can investigate your claim.

\section{OPEN ACCESS}


2 Background: Malnutrition in patients hospitalised with a stroke have been assessed using different nutritional screening methods but there is a paucity of data linking risk of

4 malnutrition to clinical outcomes using a validated tool.

5 Aims: To identify the prevalence of malnutrition risk in patients after a stroke and assess the 6 predictive value of the Malnutrition Universal Screening Tool on clinical outcomes.

7 Patients and methods: Using data from electronic records and the Sentinel Stroke National 8 Audit Programme (January 2013 and March 2016), patients aged $>18$ years with confirmed 9 stroke admitted to a tertiary care stroke unit were assessed for risk of malnutrition. The 10 association between malnutrition risk and clinical outcomes was investigated and adjusted 11 for confounding variables.

Results: Of 1101 patients, 66\% were screened at admission. Most patients ( $n=571,78.5 \%$ ) were identified as being at low risk, $4.1 \%(n=30)$ at medium risk and $17.4 \%(n=126)$ at high risk of malnutrition. Compared with low risk, patients with medium or high risk of malnutrition were more likely to have a longer hospital stay (IRR $1.30,95 \% \mathrm{Cl} 1.07,1.58$ ), and had greater risk of mortality $(10.9 \%$ versus $3.5 \%, 95 \% \mathrm{Cl} 0.03,0.13)$.

Conclusions: Prevalence of malnutrition assessed by Malnutrition Universal Screening Tool in patients after a stroke was relatively low, but nearly a third of patients were not screened. Patients classified as being at medium or high risk of malnutrition were more likely to experience negative outcomes. Early identification of this population may improve outcome if appropriate care is provided. 


\section{Introduction}

Early post-stroke complications include dysphagia, infections, recurrence of stroke, increased intracranial pressure and malnutrition. Documented prevalence of malnutrition in patients after a stroke ranges from $6.1 \%$ to $62 \%(1)$ and has been shown to affect clinical outcomes (2, 3). However, in the majority of patients, malnutrition is considered an avoidable consequence of a stroke (3). The most obvious indication of malnutrition is rapid weight loss and this pattern of weight change is recognised as an important predictor of poor outcomes (4). Available evidence indicates that, after a stroke, a reduction in weight of more than $3 \mathrm{~kg}$ both in the short and long term can impact on risk of mortality (5). Importantly, studies indicate that nutritional interventions can help improve patients' clinical outcomes if initiated early (6-8).

To provide nutritional interventions to patients at risk of malnutrition, those who are malnourished need to be identified. This can be achieved with the use of a nutritional screening tool, as recommended by national clinical guidelines for stroke which advise screening for malnutrition risk on admission and at least weekly thereafter by trained staff using a structured tool (9). A number of studies have reported on nutritional status in the stroke population using different assessment methods or screening tools $(2,6,8,10-15)$. However, a large discrepancy exists in the tools used, time of assessment and definition of malnutrition.

There is a difference between nutritional screening and nutritional assessment (16). Nutritional screening tools are designed to be used as rapid and simple methods to identify those at risk of malnutrition, while nutritional assessment involves comprehensive assessment of nutritional status by trained personnel to identify malnutrition, which can then be treated with appropriate nutritional interventions (17). The most frequently used screening tool in the UK is the Malnutrition Universal Screening Tool (MUST), a feasible way of identifying patients at risk of malnutrition in clinical and community settings (18), which has been included in the Global Leadership Initiative on Malnutrition (GLIM), a new consensus on assessment of malnutrition (19). Although MUST has been validated against nutritional assessment methods and other screening tools (20) in mixed patient populations, the evidence of its predictive value in patients after a stroke is thus far unclear. 
The paucity and inconsistency of evidence on the prevalence of malnutrition in patients after a stroke, leaves clinical practitioners with uncertainty. Therefore, the aim of this observational clinical registry and audit was to identify the prevalence of risk of malnutrition in patients after a stroke determined by MUST in a UK based secondary care facility and its association with clinical outcomes including mortality and length of stay (LoS).

\section{Materials and Methods}

This was a retrospective analysis of prospectively collected data from local Sentinel Stroke National Audit Programme (SSNAP) and the electronic patient record (EPR) at the Greater Manchester Comprehensive Stroke Centre (CSC) based at Salford Royal Foundation Trust. All patients with suspected stroke within 48-hours of onset were admitted to the stroke unit based on location of home residence. Patients with a confirmed stroke, aged at least 18 years and with a Salford postcode receiving their ongoing care at CSC were eligible for the study, and identified using Salford SSNAP data. Core SSNAP data were extracted and merged with additional data from the EPR. The clinical parameters included patients' characteristics, baseline nutritional status including weight and body mass index (BMI); MUST score at admission and before discharge; mortality, National Institutes of Health Stroke Scale (NIHSS scale), Modified Rankin Scale (mRS), complications and LoS. Ethical approval for this secondary data analysis was sought from the University of Manchester's Ethics Committee (UREC). As all data were collected as part of routine clinical care, following (proportionate) review and consideration by UREC, the study was given exemption from the requirement for ethical approval as a clinical registry, service evaluation and audit. The extraction of data was performed by the Information Technology Department at Salford Royal NHS Foundation Trust using the Electronic Patient Records (EPR). These data were matched with that of the Sentinel Stroke National Audit Programme (SSNAP) database by a member of the clinical team and fully anonymised. The research team had access to the anonymised data only. The study was formally registered as a clinical audit at the Clinical Audit Department (reference number 2016151) at Salford Royal NHS Foundation Trust.

\section{MUST score}

MUST score were collected from SSNAP data. Healthcare professionals performed MUST assessment as part of routine hospital admissions procedure and before discharge. The MUST 
methodology used incorporates three independent variables: $B M I$ score $(B M I>20.0=0, B M I$ 18.5-20.0= 1, BMI < 18.5=2); unplanned weight loss in previous 3-6 months (weight loss $<5 \%=0$, weight loss $5-10 \%=1$ and weight loss $>10 \%=2$ ); and acute disease effect score (a score of 2 was added if a patient was recently affected by a disease and there was no nutritional intake or likely to be no nutritional intake for more than 5 days). For BMI and weight loss, each variable is scored on a scale of 0 to 2 . A total sum of scores is used to categorise the risk of malnutrition as low (0), medium (1) and high ( $\geq 2)(21)$. Details on MUST score assessment can be found at the British Association for Parenteral and Enteral Nutrition website (22). We further categorised MUST into a dichotomised variable (low risk of malnutrition 0 , versus medium to high risk of malnutrition $\geq 1$ ).

\section{Statistical methods}

Standard descriptive statistics summarised the patient and clinical characteristics. Statistical inference determined the association between prevalence of risk for malnutrition and clinical outcomes, whist adjusting for potential confounders in a multiple logistic regression analysis. LoS defined as a count of the days as inpatient was modelled using a negative binomial regression to investigate the relationship between LOS and MUST. Confounders were identified prior to analysis. Weight, BMI and mRS were not included due to collinearity. The analysis was adjusted for age, gender, type of stroke, NIHSS scale and comorbidities. Further (logistic) regression modelling investigating the association between MUST and deaths in hospital, however deaths at 6-months from admission was not possible due to limited death events occurring in those with a MUST score. A Fishers exact test however was possible for death at 6 months and is reported.

\section{Results}

Between January 2013 and March 2016, records of 1,101 patients who met the eligibility criteria were extracted from EPR and combined with corresponding SSNAP data. The patient's baseline characteristics based on dichotomised MUST score for the total population are shown in Table 1, and mRS at pre-admission and discharge by MUST category is presented at Figure 1. Mean age was 73.6 (SD 13.6) years and 94\% of patients included were of a Caucasian origin with equal gender distribution. Just over half of patients ( $n=563,51.2 \%$ ) had a history 
of hypertension; about a fifth of patients ( $n=209,19 \%)$ had diabetes mellitus, atrial fibrillation $(n=197,17.9 \%)$, and a small proportion had $(n=51,4.4 \%)$ congestive heart failure. The vast majority of patients ( $n=976,89.2 \%$ ) were diagnosed with ischemic stroke, with the remainder ( $n=118,10.8 \%$ ) being diagnosed with a primary intracerebral haemorrhage. A fifth of patients had a previous transient ischemic attack $(n=218,19.8 \%)$. Based on NIHSS scale(23), no stroke symptoms (score 0) were present in $10.8 \%$ of patients, minor stroke (score 1 to 4 ) were experienced by $40.2 \%$, moderate stroke (score $5-15$ ) by $33.5 \%$, moderate to severe stroke (score $16-20$ ) by $7.3 \%$ and severe stroke (score 21 to 42 ) by $8.3 \%$ patients (Table 1 ).

\section{Table 1}

Figure 1

\section{MUST score}

A MUST score was recorded in $66 \%$ of patients both on admission and prior to discharge from hospital. On admission, the majority of patients $78.5 \%(n=571)$ were at low risk of malnutrition (MUST score 0), 4.1\% ( $n=30)$ were considered to be at medium risk (MUST score 1) and $17.4 \%$ ( $n=126)$ were classified as being at high risk of malnutrition (MUST score $\geq 2$ ). At the point of discharge from hospital, the number of patients with low risk of malnutrition increased to $85.2 \%$ ( $n=618$ ), the number of patients with medium risk increased to $5.8 \%$ $(n=42)$ and the number of patients in high risk group decreased to $9 \%(n=65)$. As expected, 38 $(90.5 \%)$ of patients with BMI between $18.5-20 \mathrm{~kg} / \mathrm{m}^{2}$ and $18(100 \%)$ of patients with BMI less than $18 \mathrm{~kg} / \mathrm{m}^{2}$ were identified as being at medium to high risk of malnutrition .

\section{Negative binomial regression}

Table 2 reports the incidence rate ratios (IRR) and corresponding 95\% confidence intervals for the adjusted association between LoS and risk of malnutrition defined by a dichotomised MUST score. Compared to low risk, medium or high risk of malnutrition was associated with an IRR $=1.30(95 \% \mathrm{Cl} 1.07$ to 1.58$)$ indicating a $30 \%$ increase in the risk of a longer hospital stay. Additionally, a linear increase in risk of a longer hospital stay was observed for NIHSS scores increasing in severity from 'moderate' through to 'severe' (IRR = 1.49, 2.64 and 3.14) when compared to no symptoms. Interestingly, minor stroke was associated with a $30 \%$ 
decrease compared to no symptoms (IRR $=0.72,95 \% \mathrm{Cl} 0.56$ to 0.93 ). Of the co-morbidities, only diabetes was associated with an increase in $\operatorname{LoS}$ of $31 \%$ (IRR $=1.31,95 \% \mathrm{Cl} 1.07$ to 1.61 ).

\section{Table 2}

\section{LoS and complications}

Median LoS for all patients was 7-days and ranged between 0 to 147 days. Twenty patients (2\%) were diagnosed with urinary tract infection in hospital. Pneumonia affected 53 patients (5.4\%). By comparison, patients who were not assessed for MUST ( $n=374)$, had a median LoS of 6-days and ranged between 0 to 147 days. Urinary tract infections were identified in 3 $(0.8 \%)$ and pneumonia in $33(8.8 \%)$ of the non-MUST assessed patients (Table 3 ).

\section{Mortality}

From the total sample of 1,101 patients, 214 (19.4\%) patients had died at 6-months follow up, and from these, 161 (14.6\%) died in hospital. From 161 patients who died in hospital, only one patient had been assessed for malnutrition with MUST score on admission. Survival of patients who died in hospital was median 3.5 (range 0-147) days. Of these, 68 (42.5\%) patients did not survive for longer than a day. For patients with a MUST $\geq 1$ (indicating a medium to high risk of malnutrition), the number of deaths was greater compared to those with a MUST= 0 (indicating a low risk of malnutrition), 17/159 (10.9\%) versus $n=20 / 571$ (3.5\%), respectively, $\mathrm{P}=0.001$. Main cause of the death at six months was recorded in 149 out of 214 patients, an ischemic stroke in $44 \%(n=66)$, spontaneous cerebral haemorrhage $16 \%(n=24)$, pneumonia in $14 \%(n=21)$, cancer $11 \%(n=16)$ and other in $15 \%(n=22)$ of patients (Table 3$)$.

\section{Table 3}

\section{Discussion}

In this observational study we examined the prevalence of risk for malnutrition in patients after a stroke and described the use of MUST as a previously validated screening tool (20) to determine future risk of malnutrition. From a total population of 1,101 patients admitted to the hospital during a three year period, only two-thirds were screened using MUST. A similar proportion of patients screened for malnutrition was previously reported by the Food Trial 
Collaboration (6). This indicates that around one third of patients with a potential risk of malnutrition are not captured by routine screening. Due to the records based dataset in the form of EPR data merged with SSNAP, we were unable to ascertain whether the reduced numbers of patients screened is related to lack of assessment or missing data.

In our sample screened for malnutrition, $21.5 \%$ of people admitted to the hospital with stroke were at moderate to high risk of malnutrition. However, due to the paucity of evidence on risk of malnutrition assessed by MUST in patients after stroke, our data can only be compared with one other study of a similar design that showed $36 \%$ risk of malnutrition (24). Surprisingly, we observed a higher proportion of patients who were at low risk of malnutrition at discharge compare to admission. This decrease (in the high risk prevalence) might reflect good nutritional care provided to patients prior to hospital discharge at our centre. A further investigation detailing the care pathway might provide more insight into observed more favourable rate of malnutrition risk.

The results of this study concur with previous data demonstrating the ability of MUST to independently predict negative outcomes in patients after a stroke (24), raising the possibility that intervening may improve clinical outcomes. Also, it was previously shown that up to one quarter of patients after a stroke became malnourished within the first week of admission, and the risk increased with prolonged hospital stay $(10,15)$. In our study, a median length of stay of 7-days was lower than 13 -days and 25 -days previously reported $(6,25)$. This was

Although the level of malnutrition in patients after admission with stroke is relatively low in comparison to other disease states (26), possibly reflecting their acute presentation, these patients still require clinical recognition and support, since malnutrition has been frequently reported as an independent prognostic factor of post-stroke complications and poor clinical outcomes $(2,14,15)$. The importance of addressing malnutrition is underlined by the cost of hospitalisation for patients with stroke, which was estimated to be up to $77 \%$ higher in patients who have a high risk of malnutrition compared to those patients who have a low risk 
support might help not only to reduce poorer clinical outcomes in patients but also reduce the burden on financial resources.

As with all retrospective studies, the main limitation of this study was the use of data collected for clinical audit and patient's records. We were limited in records availability and consequent missing data for individual outcomes. Also, the impact of dysphagia and post-stroke depression, both linked to oral intake and nutritional status were not directly factored into our analyses, which may have impacted on outcomes. Data were analysed from a single hospital in the UK and the rates of screening may reflect a degree of selection bias. Moreover the MUST score was recorded in only two thirds of patients admitted to hospital with a stroke, which limits our ability to draw strong conclusions on prevalence of malnutrition and its association with clinical outcomes. However, despite the limitations, these data provide an insight into a risk of malnutrition in a large number of participants after a stroke.

\section{Summary and Conclusion}

Our findings show that there is a significant group of patients at risk of malnutrition, and malnutrition risk as identified by MUST is associated with longer hospital stay even after adjustment for confounders. Hence, these patients require clinical recognition, and further prospective studies are warranted to evaluate any effect of nutritional interventions. Importantly, our data also show that there are substantial numbers of patients who are not screened for malnutrition on admission to hospital. These patients might be at risk of malnutrition but remain unidentified. 
249 1. Foley NC, Salter KL, Robertson J, Teasell RW, Woodbury MG. Which reported estimate of the prevalence of malnutrition after stroke is valid? Stroke. 2009;40(3):e66-74.

2. Martineau J, Bauer JD, Isenring E, Cohen S. Malnutrition determined by the patientgenerated subjective global assessment is associated with poor outcomes in acute stroke patients. Clinical nutrition. 2005;24(6):1073-7.

3. Bouziana SD, Tziomalos K. Malnutrition in patients with acute stroke. Journal of nutrition and metabolism. 2011;2011:167898.

4. Martin L, Senesse P, Gioulbasanis I, Antoun S, Bozzetti F, Deans C, et al. Diagnostic criteria for the classification of cancer-associated weight loss. Journal of clinical oncology : official journal of the American Society of Clinical Oncology. 2015;33(1):90-9.

5. Jonsson AC, Lindgren I, Norrving B, Lindgren A. Weight loss after stroke: a population-based study from the Lund Stroke Register. Stroke. 2008;39(3):918-23.

6. Food Trial Collaboration. Poor nutritional status on admission predicts poor outcomes after stroke: observational data from the FOOD trial. 2003 Jun. Report No.: 1524-4628 (Electronic) 0039-2499 (Linking) Contract No.: 6.

7. Axelsson K, Asplund K, Norberg A, Alafuzoff I. Nutritional status in patients with acute stroke. Acta medica Scandinavica. 1988;224(3):217-24.

8. Gariballa SE, Parker SG, Taub N, Castleden CM. Influence of nutritional status on clinical outcome after acute stroke. The American journal of clinical nutrition. 1998;68(2):275-81.

9. Rudd A, Bowen A, Young G, James M. National clinical guideline for stroke: 5th edition 2016. Clinical Medicine. 2017 Feb 7:69-71.

10. Davalos A, Ricart W, Gonzalez-Huix F, Soler S, Marrugat J, Molins A, et al. Effect of malnutrition after acute stroke on clinical outcome. Stroke. 1996;27(6):1028-32.

11. Davis JP, Wong AA, Schluter PJ, Henderson RD, O'Sullivan JD, Read SJ. Impact of premorbid undernutrition on outcome in stroke patients. Stroke. 2004;35(8):1930-4.

12. Chai J, Chu FC, Chow TW, Shum NC. Prevalence of malnutrition and its risk factors in stroke patients residing in an infirmary. Singapore medical journal. 2008;49(4):290-6.

13. Lim SL, Ong KC, Chan YH, Loke WC, Ferguson M, Daniels L. Malnutrition and its impact on cost of hospitalization, length of stay, readmission and 3-year mortality. Clinical nutrition. 2012;31(3):345-50.

14. Shen HC, Chen HF, Peng LN, Lin MH, Chen LK, Liang CK, et al. Impact of nutritional status on long-term functional outcomes of post-acute stroke patients in Taiwan. Archives of gerontology and geriatrics. 2011;53(2):e149-52.

15. Yoo SH, Kim JS, Kwon SU, Yun SC, Koh JY, Kang DW. Undernutrition as a predictor of poor clinical outcomes in acute ischemic stroke patients. Archives of neurology. 2008;65(1):39-43.

16. Charney P. Nutrition screening vs nutrition assessment: how do they differ? Nutrition in clinical practice : official publication of the American Society for Parenteral and Enteral Nutrition. 2008;23(4):366-72.

17. Young AM, Kidston S, Banks MD, Mudge AM, Isenring EA. Malnutrition screening tools: comparison against two validated nutrition assessment methods in older medical inpatients. Nutrition. 2013;29(1):101-6.

18. Elia M, Russell C. Nutrition screening survey in the UK and Republic of Ireland in 2011 BAPEN. 2012.

19. Cederholm T, Jensen GL, Correia MITD, Gonzalez MC, Fukushima R, Higashiguchi T, et al. GLIM criteria for the diagnosis of malnutrition - A consensus report from the global clinical nutrition community. Clinical nutrition. 2018.

20. Stratton RJ, Hackston A, Longmore D, Dixon R, Price S, Stroud M, et al. Malnutrition in hospital outpatients and inpatients: prevalence, concurrent validity and ease of use of the 'malnutrition universal screening tool' ('MUST') for adults. The British journal of nutrition. 2004;92(5):799-808. 
21. Elia M. THE 'MUST' REPORT Nutritional screening of adults: a multidisciplinary responsibility 300 Redditch: BAPEN 2003 [

301 22. BAPEN. Malnutrition Universal Screening Tool2003 May 2019; (May ). Available from: https://www.bapen.org.uk/pdfs/must/must full.pdf.

303 23. Hage V. The NIH stroke scale: a window into neurological status. NurseCom Nursing 304 Spectrum (Greater Chicago). 2011;24(15):44-9.

305 24. Gomes F, Emery PW, Weekes CE. Risk of Malnutrition Is an Independent Predictor of 306 Mortality, Length of Hospital Stay, and Hospitalization Costs in Stroke Patients. Journal of stroke and cerebrovascular diseases : the official journal of National Stroke Association. 2016;25(4):799-806. 25. Ha L, Hauge T, Spenning AB, Iversen PO. Individual, nutritional support prevents undernutrition, increases muscle strength and improves QoL among elderly at nutritional risk hospitalized for acute stroke: a randomized, controlled trial. Clinical nutrition. 2010;29(5):567-73. 26. Russell C, Elia M. Nutrition screening survey in hospitals in the UK, 2007-2011. BAPEN. 2014. 